\title{
Quantifying Damage, Saturation and Anisotropy in Cracked Rocks by Inverting Elastic Wave Velocities
}

\author{
Alexandre Schubnel ${ }^{1}$, Philip Benson ${ }^{1,2}$, Ben Thompson ${ }^{1,3}$, Jim Hazzard $^{1,4}$ and \\ R.Paul Young ${ }^{1}$
}

\begin{abstract}
Abbreviated title:
DAMAGE, SATURATION AND ANISOTROPY IN CRACKED ROCKS
\end{abstract}

\section{Keywords:}

Elastic wave velocities, Anisotropy, Crack density, Saturation, Effective medium, Attenuation.

submitted for a special issue of Pure and Applied Geophysics

contact:

alexandre.schubnel@utoronto.ca

\footnotetext{
${ }^{1}$ Lassonde Institute, University of Toronto, 170 College street, Toronto, ON M5S 3E3, Canada

${ }^{2}$ Mineral, Ice and Rock Physics Laboaratory, University College London, Gower Street, London WC1E 6BT, UK

${ }^{3}$ Applied Seismology Laboratory, Department of Earth Sciences, Liverpool University , 4 Brownlow St., Liverpool L69 3GP UK

${ }^{4}$ Rocsciences Inc., 439 University Ae., Toronto, ON M5G 1Y8, Canada
} 
Abstract

Theoretically, crack damage results in a decrease of elastic wave velocities and in the development of anisotropy. Using non-interactive crack effective medium theory as a fundamental tool, we calculate dry and wet elastic properties of cracked rocks in terms of a crack density tensor, average crack aspect ratio and mean crack fabric orientation using the solid grains and fluid elastic properties. Using this same tool, we show that both the anisotropy and shear wave splitting of elastic waves can be derived. Two simple crack distributions are considered for which the predicted anisotropy depends strongly on the saturation, reaching up to $60 \%$ in the dry case. Comparison with experimental data on two granites, a basalt and a marble, shows that the range of validity of our model extends up to total crack density of approximately 0.5 , considering symmetries up to orthorhombic. In the isotropic case, Kachanov's [1994] non-interactive effective medium model was used in order to invert elastic wave velocities and infer both crack density and aspect ratio evolutions. Inversions are stable and give coherent results in term of crack density and aperture evolution. In the anisotropic cases - both transverse isotropic and orthorhombic symmetries were considered - anisotropy and saturation patterns were well reproduced by the modelling and mean crack fabric orientations is recovered. Inversion results agree very well with the laboratory data and are consistent with the rock microstructure in the different rocks investigated. Our results point out that: (1) it is possible to predict damage, anisotropy and saturation in terms of a crack density tensor and mean crack aspect ratio and orientation; (2) Using well constrained laboratory 
data and wave velocity inversion, it is possible to extrapolate the contemporaneous evolution of crack density, anisotropy and saturation using wave velocity inversion as a tool. 


\section{Introduction}

Under crustal conditions, all rocks contain cracks. However, despite the fact that cracks generally represent only small amounts of porosity, they can exert considerable influences upon the rock bulk mechanical and transport properties (Simmons and Brace, 1965; Walsh 1965, Brace et al. 1968; Simmons et al., 1975). For example, the existence of embedded microcrack fabrics in rocks contributes to the difference between static and dynamic elastic moduli (Mavko and Jizba 1991), may significantly influence the fracture toughness (Nasseri et al. 2005) and aligned crack fabric may produce elastic anisotropy (Kern 1978, Kern et al. 1997). When fluids are present, cracks also play a key role in making the rock permeable on a macroscopic scale. In the oil industry, seismic data and velocity field analysis are used on a daily basis to quantify the oil content and the identify fluid properties, i.e. oil or gas. In seismotectonics, an open question is to know whether earthquakes and volcanic eruptions can be predicted from elastic wave velocity variations, which are possible time dependent precursory effect (Chun et al. 2004; Gao and Crampin 2004). The understanding and quantification of rock elastic wave velocity variations is of great interest to extract from seismic and seismological data any information on the physical state of the rock. For example, it has major implications when considering the possible hydro-mechanical coupling taking place during the seismic cycle (Miller 2002), forecasting the life time of oil reservoirs, or the integrity of underground storage of hazardous wastes.

In the last thirty years, a large number of models aiming to predict properties 
of materials as a function of damage have been developed (O'Connell and Budiansky 1974, 1977; Cheng and Toksoz 1979; Hudson 1981, 1982, 1986, Nishizawa 1982, Kachanov 1994, Sayers and Kachanov 1991, 1995, Le Ravalec and Guéguen 1996) in the framework of Effective Medium Theories (EMT), most of which are based on first order perturbation theory. Statistically, the effective elastic properties of an initially isotropic medium depend on a few intrinsic parameters:

- the solid matrix elastic properties (Young's modulus $E_{o}$ and Poisson ratio $\left.\nu_{o}\right)$

- the fluid bulk modulus $K_{f}$

- the crack density $\rho$ defined as $\rho=\frac{1}{V} \sum^{N} c_{i}^{3}$ where $c_{i}$ is the radius of the ieth crack, $N$ being the total number of cracks embedded in the Representative Elementary Volume (REV) $V$.

- the crack geometry (in our case, we consider penny shaped geometry (figure 1a) and their average aspect ratio $\zeta=\langle w / c\rangle$.

In an isotropic matrix containing a random distribution of cracks, the effective elastic modulus of a rock $M^{*}$ is a linear function of the crack density that can be written in the form (first perturbation order):

$$
\frac{M_{o}}{M^{*}}=1+h \cdot \rho
$$

where $M_{o}$ is the slid matrix elastic modulus and $h$ is a positive scaling parameter that depends on the matrix and fluid properties, the geometry of the cracks and the interactions between them. The scalar $h$ has been calculated by Eshelby 
[1957] and various following authors for all kind of inclusion geometries (spherical, elliptical, penny-shaped discs, rectangular plates or linear cracks) and fluid properties. One can find a good compilation in Mavko et al. [1998]. The most simple and non-controversial model type is non-interactive effective medium theory because it is independent of crack centers distribution, which was shown to be valid when cracks are distributed randomly or aligned (Kachanov 1994, Sayers and Kachanov 1995, Guéguen and Schubnel 2003). We report here an approach based on Kachanov (1994) non-interactive EMT scheme which allows the relatively straightforward extraction of crack density, aspect ratio and alignment from elastic wave velocity measurements made on several rock types in the laboratory. As the extensive laboratory input data compiled in this study were measured independently, our study provides an a ideal opportunity to test and investigate the model applicability.

\section{Effective elastic medium containing cracks: non- interactive formulation}

When neglecting stress interactions between cracks, the effective elastic modulii of a cracked solid can be calculated exactly and rigorously in a unique manner that depends solely upon the crack orientations and distribution. Such hypothesis is often wrongly confused with the low crack density approximation. Indeed, for certain distributions where interactions are compensating geometrically such as a random (isotropic) distribution or aligned cracks, it has been shown that 
the non-interactive approximation is valid at least up to crack densities of 0.5 (Kachanov 1994; Sayers and Kachanov 1995; Schubnel and Guéguen 2003). In the non-interactive approximation, each crack is considered to be isolated and the elastic perturbation in compliance due to cracks $\Delta S_{i j k l}$ is simply the sum of each single crack contribution. Since the average bulk elastic strain is the sum of the matrix elastic strain and the superposition of elastic strain of each individual crack, effective elastic modulii can simply be calculated using the elastic potential $f$ (Kachanov 1994; Sayers and Kachanov 1995).

\subsection{Isotropically distributed cracks}

In the isotropic case, Kachanov [1994] showed that the effective Young $E^{*}$ and shear modulii $\mu^{*}$ of a rock could be written as:

$$
\left.\frac{E_{o}}{E^{*}}=1+\frac{\rho}{3 h}\left(1+\frac{3}{5}\left[\left(1-\frac{\nu_{o}}{2}\right) \frac{\delta}{1+\delta}-1\right)\right]\right)
$$

and:

$$
\left.\frac{\mu_{o}}{\mu^{*}}=1+\frac{\rho}{3 h}\left(1+\frac{2}{5}\left[\left(1-\frac{\nu_{o}}{2}\right) \frac{\delta}{1+\delta}-1\right)\right]\right)
$$

where $h$ is a geometrical factor linked to the penny-shaped geometry (see appendix I) and given by,

$$
h=\frac{16\left(1-\nu_{o}^{2}\right)}{3 \pi\left(1-\nu_{o} / 2\right)}
$$

$\delta$ characterizes the coupling between the stress and the fluid pressure, and determines the fluid impact on the effective crack compliance:

$$
\delta=\left(1-\nu_{o} / 2\right) \frac{E_{0} \zeta h}{K_{f}}
$$


$\delta$ is a non-dimensional number that compares the fluid bulk modulus to the crack bulk modulus $\left\{\left(1-\nu_{o} / 2\right) E_{o} \zeta h\right\}$ assuming that all change in the crack volume is due to aperture variations (see appendix I). As a lower bound, i.e. for an incompressible fluid such as water, and using $E_{0} \sim 5.10^{10}, \delta \rightarrow 0$ and is therefore negligible if $\zeta$ is small $\left(<10^{-3}\right)$, as expected for a crack. As an upper bound, i.e. for a compressible fluid such as dry air, $\delta$ is much greater than 1 and $\frac{\delta}{1+\delta} \rightarrow 0$.

Figure $2 \mathrm{a}$ and $2 \mathrm{~b}$ presents the normalized $\mathrm{P}$ and $\mathrm{S}$ wave velocity isocontours respectively as a function of both crack density and the aspect ratio. The velocities were calculated from equations 2-3 using solid matrix and fluid elastic parameters typical of a water saturated granite and equal to: $E_{o}=85 G P a$, $\nu_{o}=0.25$ and $K_{f}=2 G P a$. Velocities were normalized to the crack free velocities (i.e. $\rho=0$ ) to remove bulk density effects. On figure $2 \mathrm{a}$, the $\mathrm{P}$ wave velocity is shown to decrease with crack density. However, at a given crack density, $\mathrm{P}$ wave velocity remains more or less constant with aspect ratio. On the contrary, whilst $\mathrm{S}$ wave velocity also decreases with crack density on figure $2 \mathrm{~b}$, they are also very sensitive to the crack mean aspect ratio. Figure $2 \mathrm{c}$ shows that the evolution of the $\mathrm{Vp} / \mathrm{Vs}$ ratio is then a function of both crack density and aspect ratio when cracks are saturated with fluid. This way, Kachanov [1994] non-interactive model permits the quantification of both the crack density $\rho$ and the aspect ratio $\zeta$, through the saturation coefficient $\delta$ (equation 5 ). It is a direct consequence that when measuring $\mathrm{Vp}$ and $\mathrm{Vs}$ in saturated rocks containing cracks, one can expect therefore to be able to extract from those measurements 
not only the crack density, but also the aspect ratio.

\subsection{Anisotropic formulations}

In the general most case of an isotropic matrix containing penny shaped cracks, the additional elastic compliance due to those $\boldsymbol{\Delta} \mathbf{S}_{\mathbf{i j k l}}$ can be expressed as (Sayers and Kachanov 1995):

$$
\Delta S_{i j k l}=\frac{1}{4 h E_{o}}\left(\delta_{i k} \alpha_{j l}+\delta_{i l} \alpha_{j k}+\delta_{j k} \alpha_{i l}+\delta_{j l} \alpha_{i k}\right)+\beta_{i j k l}
$$

where $\alpha_{i j}$ is the crack density tensor :

$$
\alpha_{i j}=\rho .<n_{i} n_{j}>
$$

and $\beta_{i j k l}$ the saturation tensor:

$$
\beta_{i j k l}=\rho \cdot\left[\left(1-\frac{\nu_{0}}{2}\right) \frac{\delta}{1+\delta}-1\right]<n_{i} n_{j} n_{k} n_{l}>
$$

$\delta_{i j}$ is the Kronecker symbol and we recall that the scalar (or total) crack density is equal to $\rho=\operatorname{tr}(\alpha)=N c^{3} / V$. In the case of an orthorhombic crack distribution, the crack density tensor is diagonal, and it follows from equations (6-8) that the 9 independent effective elastic compliances can be expressed:

$S_{i j k l}=\left\{\begin{array}{c}\left.=S_{i j k l}^{o}+\frac{\rho}{E_{o} h}\left(<n_{i}^{2}>+\left[\left(1-\frac{\nu_{0}}{2}\right) \frac{\delta}{1+\delta}-1\right)\right]<n_{i}^{4}>\right) \text { if }\{i=j=k=l\} \\ \left.=\quad S_{i j k l}^{o}+\frac{\rho}{E_{o} h}\left(\frac{<n_{i}^{2}>+<n_{j}^{2}>}{4}+\left[\left(1-\frac{\nu_{0}}{2}\right) \frac{\delta}{1+\delta}-1\right)\right]<n_{i}^{2} n_{j}^{2}>\right) \text { if }\{i=k \text { and } j=l\} \\ =\quad \\ \left.S_{i j k l}^{o}+\frac{\rho}{E_{o} h}\left[\left(1-\frac{\nu_{0}}{2}\right) \frac{\delta}{1+\delta}-1\right)\right]<n_{i}^{2} n_{k}^{2}>\text { if }\{i=j \text { and } k=l\}\end{array}\right.$ 
Recalling the relationship between dependent constants, the formulation for transverse isotropy can also be obtained from equation 9. Assuming now a continuous distribution function of crack orientations, the average ith component of the crack fabric can be defined the following way :

$$
<n_{i}>=\frac{1}{2 \pi} \int_{0}^{2 \pi} d \phi \int_{0}^{\pi / 2} \psi(\theta, \phi) n_{i} \sin \theta d \theta
$$

$\psi(\theta, \phi)$ being the orientation distribution function (cf. figure 1a). In the same way, the tensors $<n_{i} n_{j}>$ and $<n_{i} n_{j} n_{k} n_{l}>$, which represent respectively the second order and the fourth order moments of the crack orientation distribution function respectively, can be calculated easily.

\subsection{Wet and dry elastic waves anisotropy: numerical re- sults}

For the sake of simplicity, we will consider Dirac distributions, so that $\left.<n_{i}\right\rangle=$ $n_{i}$ and subsequently, in such a way that the average crack fabric orientation is described as on figure 1a for a single crack. In this section, we consider two different types of crack distributions: i) radial cracks $\left(n_{3}=0, n_{1}=n_{2}=\right.$ $1 / \sqrt{2})$ and ii) planar cracks $\left(n_{3}=1, n_{1}=n_{2}=0\right)$. The first case corresponds to a distribution of symmetrically distributed radial cracks (symmetry along the vertical axis, cracks normal in the horizontal axis) and is representative of the TI symmetry (figure 1b). The second case corresponds to horizontally aligned coplanar penny shaped cracks and is representative of both TI and orthorhombic symmetry (figure 1c). Furthermore, only the lower and upper 
bound of saturation, i.e. for an incompressible fluid such as water $\delta \rightarrow 0$ and for a compressible fluid such as dry air, $\frac{\delta}{1+\delta} \rightarrow 0$, were investigated. In all that follows, the solid matrix Young's modulus and Poisson ratio were taken as equal to the ones of an isotropic and crack free granite material, i.e. $E_{o}=85 \mathrm{GPa}$ and $\nu_{o}=0.25$ respectively.

\subsubsection{Radial cracks}

The case of radial cracks is illustrated on figure 3 . Figure $3 \mathrm{a}$ and $3 \mathrm{~b}$ show the normalized $\mathrm{P}$ velocities versus the angle of propagation $\gamma$ of the elastic wave, in wet and dry conditions respectively, and for crack densities equal to $0.1,0.25,0.5$ and 1. The angle $\gamma$ is defined as that between the vertical axis $O x_{3}$ and the wave vector. For example, elastic waves propagating at 0 and 90 degrees correspond to propagation along the vertical axis and horizontal axis respectively. The normalized wave velocity is the ratio of $V_{\gamma}$ to the solid grain velocity. In the case of radial cracks, $\mathrm{P}$ waves propagating vertically are unaffected and do not "see" the cracks. Anisotropy is larger in the dry case (up to $50 \%$ in the dry case, up to $\sim 40 \%$ in the wet case) but the pattern is very comparable in both cases. Figures $3 \mathrm{c}$ and $3 \mathrm{~d}$ show the results obtained in term of the shear wave splitting (or birefringence between vertically (SV) and horizontally (SH)

polarized S waves : $\left.\frac{S V(\gamma)-S H(\gamma)}{S V(\gamma)} * 100\right)$, in the wet and dry cases respectively. In the dry case, SV waves always travel faster than SH. In the wet case, maximum splitting is observed at a $\gamma$ angle of 60 degrees, whereas maximum dry splitting is obtained for horizontally propagating $\mathrm{S}$ waves. However and as expected for 
a set of radial cracks, the effect of fluid is rather small.

\subsubsection{Planar cracks}

Figure 4 shows numerical results obtained in the case of planar cracks. Figure 4a and $4 \mathrm{~b}$ show the normalized $\mathrm{P}$ velocities as a function of propagation angle $\gamma$ and crack density in the wet and the dry cases respectively. As noted earlier, the effect of cracks is much stronger in the dry case than in the wet case. This time, cracks are "invisible" to waves propagating in the horizontal direction $\left(\gamma=90^{\circ}\right)$; and in the dry case (figure $\left.4 \mathrm{~b}\right)$, the $\mathrm{P}$ wave velocity decrease is very large (up to $60 \%$ ) for waves propagating vertically which is expected from geometrical considerations. However, and because the fluid is assumed to be fully incompressible, cracks are also "invisible" in the direction $\gamma=0$ degrees in the wet case (figure 4a). This can be explained theoretically as in Kachanov's model, the normal crack compliance is assumed to be equal to zero (see Appendix I) when the fluid is fully incompressible (or when the crack is fully constrained). Therefore, in the wet case, the maximum anisotropy (up to $20 \%$ for $\rho=1$ ) is seen for waves propagating at 45 degrees from the vertical, for which the associated shear strain is maximum. Figures $4 \mathrm{c}$ and $4 \mathrm{~d}$ show the predicted results for shear wave splitting $\left(\frac{S V(\gamma)-S H(\gamma)}{S V(\gamma)} * 100\right)$. Again, the pattern is very different in the wet and the dry cases. As expected, in the dry case SH waves always propagate faster than SV waves. In the wet case however, and for propagation angles between 0 and 60 degrees, SV wave are faster than $\mathrm{SH}$ waves. Conversely, SH wave velocity is higher than SV wave velocity for angles 
larger than 60 degrees. The shear wave splitting maximum observed is thus also an effect of fluid incompressibility.

In each of the above crack distributions, the non-interactive model shows a clear difference between dry and fully saturated cracks, both for $\mathrm{P}$ waves and $\mathrm{S}$ waves. Intermediate results for partial saturation could be obtained considering the $\delta$ parameter and average crack aspect ratio. It shows that, to a certain extent, the $\mathrm{P}$ wave anisotropy and $\mathrm{S}$ wave birefringence patterns can correspond to a genuine crack distribution and saturation state. Therefore, combining velocity data on $\mathrm{P}$ wave anisotropy and $\mathrm{S}$ wave birefringence should allow to investigate the "average" crack distribution in a rock, as well as the saturation state. Unfortunately, such kind of extensive wave velocity data is very rare in the literature. Such data exists at the field scales, but one would need to take into account the dispersion effects due to frequency and "squirt flow" (Mavko and Nur 1975). This may be done using Biot-Gassman or Brown and Korringa's equations [1975], as in Schubnel and Guéguen [2003].

\section{Inversion of experimental data}

In the laboratory, experimental studies measuring the evolution of dynamic elastic properties under conditions simulating upper crust burial depths can be performed thanks to the use of piezoelectrics transducers (PZT), which eigen frequency are generally well into the dynamic range $(>100 \mathrm{KHz})$. Despite the experimental difficulties, such studies have been undertaken and can provide 
'well-constrained' experimental datasets which are perfectly suited to attempt predicting the evolution of rock fabric parameters such as crack density, aspect ratio and alignment.

When modelling the evolution of elastic velocities, we restricted ourselves to the case of an initially isotropic solid rock matrix. The crack distribution function obeyed either an isotropic, transverse isotropic or orthorhombic symmetry. In such conditions, the effective elastic properties predicted by Kachanov's model [1994] and equations (2, 3 and 9) are dependent only upon the matrix Young's modulus $E_{o}$ and Poisson ratio $\nu_{o}$, and more importantly, the scalar crack density $\rho$. Additionally, when cracks are non-randomly oriented, elastic wave velocities also depend on the mean orientation $(\theta, \phi)$ of the crack fabric with respect to the axis of symmetry. Finally, when saturated, elastic properties also depend on the saturation coefficient $\delta$ and therefore the average crack aspect ratio $\zeta$ and fluid bulk modulus $K_{f}$. In the following, we performed simple least square inversions of laboratory data sets:

$$
R M S_{i}=\frac{1}{N_{i}} \sum_{N_{i}}\left(V_{\gamma}^{\text {data }}-V_{\gamma}^{\text {model }}(\rho, \zeta, \theta, \phi)\right)^{2}
$$

in order to recover the crack density, average crack aspect ratio and orientation. Inversions are performed using initial P and S wave velocities of the "uncracked" material as calculated from experimental and/or petrological considerations, thus reflecting the average bulk solid matrix elastic properties. Model elastic wave velocity field was calculated from equations (2, 3 and 9$)$ as a function of $\rho, \zeta$ when the rock was saturated with water together with $\theta$ and $\phi$ when the rock was anisotropic. Each modeled velocity fields are then compared using a 
simple RMS technique (equation 10) with experimental measures. In this equation, $N_{i}$ represents the number of $\mathrm{P}$ and $\mathrm{S}$ velocity measurements along several $\gamma$ directions at each pressure step $i$ of a given experiment. The lowest error between modeled and data velocity fields is taken as being the best inversion result, which outputs the quadruplet $(\rho, \zeta, \theta, \phi)$. The agreement between data and best fit velocities is, in general, very good with average error between model and data points lower than $0.05 \mathrm{~km} / \mathrm{sec}$ which clearly demonstrates the stability of the inversion, as a direct consequence of the well constrained laboratory data itself.

\subsection{Isotropic inversions}

Elastic wave velocities have been widely shown to increase with increasing hydrostatic pressure, because of crack closure. Conversely and in the presence of shear stress, elastic wave velocities can decrease due to nucleation and propagation of new microcracks. In the following, we compile experimental results obtained on two different rock types, representative of contrasting isotropic microcrack fabric, a porphyritic alkali basalt from Mount Etna and Carrara marble.

\subsubsection{Crack density and aspect ratio evolution as a function of hy- drostatic pressure}

In the case of the Etnean basalt, the simultaneous evolutions of $\mathrm{P}$ and $\mathrm{S}$ wave elastic wave velocity were measured during hydrostatic compression of 3 different 
rock samples (38.1mm diameter by $40 \mathrm{~mm}$ length) cored in three orthogonal directions (figure 5a). The measurements, performed in a high pressure confining cell installed at University College London (Benson 2004), showed no marked elastic anisotropy in the rock. The experimental P-wave velocities ranged from $5.35 \pm 0.13 \mathrm{~km} / \mathrm{s}$ at $5 \mathrm{MPa}$ to $5.88 \pm 0.12 \mathrm{~km} / \mathrm{s}$ at $80 \mathrm{MPa}$; while S-wave velocities ranged from $3.30 \pm 0.04 \mathrm{~km} / \mathrm{s}$ to $3.60 \pm 0.04 \mathrm{~km} / \mathrm{s}$. P and $\mathrm{S}$ elastic were inverted using equations (2) and (3). Crack free elastic wave velocities were taken as equal to $V_{p}=6400 \mathrm{~m} / \mathrm{s}$ and $V_{s}=3750 \mathrm{~m} / \mathrm{s}$ (i.e. solid matrix elastic parameter equal to $E=100 G P a$ and $\nu=0,22)$. The fluid bulk modulus was taken as $K_{f}=2 G P a$.

Figure 5a compares the model $\mathrm{P}$ and $\mathrm{S}$ elastic velocities (solid and dashed line) to the experimental results (symbols). Because the degree of freedom of the inversion is zero (i.e. $\mathrm{P}$ and $\mathrm{S}$ wave velocities were modeled using only two distinct parameters: crack density and aspect ratio), the fit appears perfect visually, well below the experimental error bar. Figure 5b shows the error contour maps between modeled and data velocities as a function of crack density and aspect ratio for sample EBD01 at $80 M P a$ respectively. As illustrated, the inversion is stable as only one minima can be distinguished and for a wave velocity doublet $(\mathrm{P}, \mathrm{S})$ corresponds a unique solution for the crack density and aspect ratio doublet $(\rho, \zeta)$. As an output, the model gives back the evolution of parameters $(\rho, \zeta)$ with confining pressure which are displayed on figures $6 \mathrm{a}$ and $6 \mathrm{~b}$. Result from the inversion shows a decrease in crack density from $\sim 0.5$ to $\sim 0.35$ during pressurization, while the average crack aspect ratio decreases 
by one order of magnitude approximately. The recovered evolution of aspect ratio, which dropped severely within the first MPa's, is thus consistent with that expected intuitively, i.e. elastic crack closure. As displayed on figure 2c, the model interprets not only the increase in both $\mathrm{P}$ and $\mathrm{S}$ velocities, but also the fact that the ratio $\mathrm{P} / \mathrm{S}$ is changing. This is probably one of the key advantages in using Kachanov's [1994] scheme when modelling elastic properties in isotropy.

\subsubsection{Crack density and aspect ratio evolution as a function of shear stress}

Carrara marble is a well investigated marble, with coarse grainsize $(150 \mu m)$ and a very low initial anisotropy $(<1 \%)$. Schubnel et al. [2005] measured both $\mathrm{P}$ and $\mathrm{S}$ wave velocities during a full tri-axial cycle in wet conditions $(P c=260 M P a$ and $P p=10 M P a)$. Initial $\mathrm{P}$ wave velocity was equal to $5.9 \mathrm{~km} . \mathrm{s}^{-1}$ while final $\mathrm{P}$-wave velocity was lower than $3 \mathrm{~km} . \mathrm{s}^{-1}$. Figure $7 \mathrm{a}$ shows the evolution of both $\mathrm{P}$ and $\mathrm{S}$ wave velocities as a function of effective mean stress $\mathbf{P}=\left[\left(\sigma_{1}+2 \sigma_{3}\right) / 3-P_{p}\right]$. In the first phase, velocities increase due to crack closure. When the onset of crack propagation is reached, wave velocities begin to decrease rapidly due to damage accumulation. During the subsequent relaxation period, wave velocities increase again, before decreasing drastically due to stress relief microcracking as hydrostatic stress is removed. On the figure, the fit between data and modelled velocity appears perfect for the same reasons as on figure 5a. This time, the model crack-free parameters were taken as that of a non-porous calcite aggregate, i.e. $E=100 G P a$ and $\nu=0.32$. Figure $7 \mathrm{~b}$ 
shows the evolution of crack density (solid diamonds) and aspect ratio (open squares) as a function of effective mean stress. During the first phase, crack density drops from 0.35 to 0.2 while aspect ratio drops from 0.01 to lower than 0.001 (more than one order of magnitude) which clearly shows crack closure. During the second phase, crack density increases slightly to 0.5 while aspect ratio increases rapidly to 0.5 , showing that high aspect ratio voids are being opened, which is consistent with the microstructure and the deformation mode in the cataclastic regime (e.g. Fredrich et al. 1989; Schubnel et al. 2005). During the relaxation phase, crack density remains more or less constant while the aspect ratio decreases. This might be a clear geophysical signature of viscoelastic crack closure and aperture reduction as mentioned by Beeler and Tullis [1997]. The fourth phase corresponds to a rapid increase in both crack density and aspect ratio due to stress relief and crack opening. It is interesting to note that the final crack density is larger than 1 , although no macroscopic rupture or strain localization band was observed.

\subsection{Anisotropic inversions}

Most rocks are characterized by anisotropic crack pattern, often due produced by deviatoric stress fields. For example, as a rock body is being deformed during triaxial compression experiments, cracks grow and propagate along preferential orientations, leading to an overall anisotropic elastic pattern (Hadley 1975; Schubnel et al. 2003). However and in the following, because the upperlimit of a non-interactive model is obviously that of instable crack propagation, 
we do not pretend to deal with coalescence or rupture propagation, but with phenomena prior to these.

\subsubsection{Crack density as a function of depth and hydrostatic pressure}

Granodiorite samples retrieved from the Nojima fault core were investigated experimentally at room pressure by Zamora et al. [1999]. Elastic wave velocities, measured in the laboratory $(500 \mathrm{KHz})$, are in good agreement with the sonic log performed during drilling (10KHz, Zamora et al. 1999). In both dry and wet conditions, $\mathrm{P}$ wave velocities were measured along the vertical axis and along 18 different directions in the horizontal plane; $\mathrm{S}$ wave velocities propagating along the vertical axis were measured along 18 different horizontal polarizations. Figure 8 presents the experimental data (symbols) obtained on 4 different samples, retrieved at 220,232, 362 and 429 meters respectively. Wet velocities are marked by plain symbols, while dry velocities are marked by empty symbols. For each of these samples, a simultaneous inversion of the dry and wet elastic wave velocity field was performed using equations 9 and 10. Crack free elastic parameters were taken as equal to that of sample $232\left(E_{o}=85 G P a\right.$ and $\left.\nu_{o}=0.25\right)$, which presented the least amount of damage in the column. The inversion was performed simultaneously on both dry $\left(\frac{\delta}{1+\delta} \rightarrow 0\right)$ and saturated $(\delta \rightarrow 0)$ data. Modeled velocities are represented on figure 8 as solid and dashed lines. It shows that Kachanov [1994] model is able, at a given crack density, to effectively predict both the observed anisotropy pattern and the saturation effects. In particular, the agreement between the modeled velocity field and the laboratory data is 
excellent, at least up to $\rho=0.4$ (sample 429). A crack density of 0.2 for sample $220,0.125$ for sample $362,0.4$ for sample 429 was calculated. If the dip of the distribution is not well constrained due to the paucity of vertical measurements (and the absence of diagonal measurements - see figure 3 and 4), it is nevertheless in overall agreement with the geological setting (Schubnel, 2002). In addition, the azimuth of the crack fabric is well constrained and was found to be more or less constant in the column. Unfortunately, it was not possible to compare that "experimental" azimuth to the regional setting, due to the lack of orientation on the samples. However, the azimuth appears constant throughout the column and this is what would be intuitively expected in a fault zone, giving us additional confidence in the modelling study.

\subsubsection{Crack density and aspect ratio evolution as a function of shear stress}

Finally, we present $\mathrm{P}$ wave velocities measurements along several directions during a tri-axial compression in dry conditions $(P c=50 \mathrm{MPa})$ on a sample Westerly granite. The experiment was performed at the USGS at Menlo Park (Thompson et al. 2003). Initial $\mathrm{P}$ wave velocity field was isotropic and equal

to $5.9 \mathrm{~km} \cdot \mathrm{s}^{-1}$ while final $\mathrm{P}$ wave anisotropy was larger than $30 \%$, which is consistent with observations performed by Schubnel et al. 2003 during triaxial compression of Oshima granite. Despite the non-interactive crack model being less relevant for experiments investigating crack propagation, it nevertheless 
provides a useful tool to study and quantify the first phase of crack propagation, which is stable in triaxial compression experiments. Figure 9 presents $\mathrm{P}$ wave velocity data (symbols) as a function of raypath angle and shear stress steps. Modelled velocities are represented by solid and dashed lines. Model crackfree parameters were taken as $E=85 G P a$ and $\nu=0.25$. Data and model velocities fit well, and the anisotropic pattern is very similar to that of radial cracks presented on figure 3 , as you would expect during the primary phases of a triaxial compression experiment. The orientation of the crack fabric is first vertical and then appears to continuously diminish: at $617 \mathrm{MPa}$, the mean crack fabric dip is $78^{\circ}$ with respect to horizontal, while the crack density is shown to increase up to 0.44 . Nevertheless, the quality of the inversion, although poorly constrained (only $\mathrm{P}$ waves) is stable and coherent. The $\mathrm{P}$ wave anisotropy pattern, is once again, very well reproduced.

\section{Discussion and conclusions}

For validation, we compare our model to that of a numerical simulation of damage evolution using the Particle Flow Code in 3 Dimensions (Itasca Consulting Group). First, a laboratory study was conducted in which a $50 \mathrm{~mm}$ cubic sample of Crossland Hill sandstone was subjected to true triaxial loading with velocity measurements taken parallel to each of the principal stress directions. The experiment was performed at Imperial College London (King 2002) and then simulated with a distinct element modelling approach using PFC3D. In the nu- 
merical experiment, the sample of Crossland Hill sandstone was simulated by an assemblage of 20,000 spherical particles closely packed and bonded together at points of contact. Particle stiffnesses and bond strengths were set such that the macro stiffness and strength of the model matched that of the actual rock. The numerical model and the actual rock sample were both subjected to two episodes of hydrostatic loading up to $100 \mathrm{MPa}$ and then deviatoric loadings. Results of the numerical and laboratory study can be found in Hazzard and Young (2004). The model is fully dynamic so that changes in wave velocities can be measured with changes in stress. The number of 'cracks' in the model was then directly counted. Figure 10 compares the crack densities calculated from measured velocities and using equations (2-3), compared with the crack densities calculated by directly counting the number of cracks (or broken bonds) in the numerical assemblage. To arrive at the curve for 'direct counting', all cracks were assumed to be closed at $100 \mathrm{MPa}$ hydrostatic stress and the number of particle-particle contacts and bonds broken relative to this stress state were counted as cracks. Moreover, cracks were assumed to have a radius equal to the particle size. It is clear that there is a fairly good match between the directly counted and calculated crack densities, which validates the modelling. However, the direct counting seems to systematically overestimate the crack density. This is probably due to the fact the crack radius are overestimated when taken as equal to the particles radius. Hence, deviation between theoretical and numerical modelling increases with increasing crack density. This may also reflect the fact that the material is no longer isotropic and that a Transversely Isotropic 
formulation might produce better results.

In conclusion, by using non-interactive crack effective medium theory as a fundamental tool, it is possible to calculate the cracked rock dry and wet elastic properties in terms of a crack density tensor, average crack aspect ratio and mean crack fabric orientation using the solid grains and fluid elastic properties solely. Using the same method, both the anisotropy and shear wave splitting of elastic waves can be derived. Two simple crack distributions have been considered for which the predicted anisotropy depends strongly on the saturation, reaching up to $60 \%$ in the dry case.

Comparison with available experimental data shows that the range of validity of non interactive crack theory extends up to at least a total crack density of 0.5 and to symmetries up to orthorhombic. In the isotropic case, Kachanov's [1994] model was used in order to invert elastic wave velocities and infer both crack density and aspect ratio evolutions. Inversions results were stable and coherent in term of crack density and aperture evolutions. In the anisotropic cases, anisotropy patterns were well reproduced by the modelling allowing the mean crack fabric orientations to be recovered. Inversion results agreed very well with the data and were consistent with the microstructure of the different rocks investigated here. It need to be pointed that for crack densities larger than 0.5 , the predictions of the model are less accurate but would nevertheless remain within the same physical trend, which is a major difference with the Self-Consistent method (O'Conell and Budiansky 1975). A natural extension of 
this work would be to try modelling permeability evolutions from velocity data, as attempted by Benson et al. 2005 and/or incorporate into the inversion the porosity data.

In summary, our results illustrate that: (1) it is possible to predict anisotropy in terms of a crack density tensor and mean aspect ratio using non-interactive crack theory, (2) using "well constrained" laboratory data, it is possible to investigate crack density, anisotropy and saturation using wave velocity inversion. Unfortunately, such kind of extensive wave velocity data is very rare in the literature.

\section{Acknowledgements}

The authors would like to acknowledge Dr. Maria Zamora, Dr. Anne Marie Boulier and Prof. Philippe Pezzard for providing the data on the Nojima fault core. Dr. Sergio Vinciguerra, Concetta Trovato and Professor Philip Meredith provided the UCL data and should be thanked. Dr. Luigui Burlini and Jérôme Fortin helped acquiring the data on Carrara marble. This work was developed thanks to many useful discussions with Prof. Mark Kachanov and Prof. Yves Guéguen. Additional and useful comments were provided by Dr. David Collins and Dr.M.H.B. Nasseri. Finally, this study was supported by an NSERC discovery grant obtained by Prof. R.Paul Young. 


\section{References}

Beeler, N.M. and Tullis, T.E. (1997), The roles of time and displacement in velocitydependent volumetric strain of fault zones, JGR, 102, 22595-22609.

Benson, P. M. (2004), Experimental study of void space, permeability and elastic anisotropy in crustal rock under ambient and hydrostatic pressure, Ph.D. thesis, 272pp., University of London, London.

Benson P., Schubnel A., Vinciguerra S., Trovato C., Meredith p. and Young R.P. (2005), Modelling the permeability evolution of micro-cracked rocks from elastic wave velocity inversion at elevated hydrostatic pressure, submitted to JGR.

Brace, W.F., Walsh, J.B., and Frangos, W.T. (1968), Permeability of granite under high pressure, JGR, 73, 2225-2236.

Brown, R., and Korringa, J. (1975), On the dependence of the elastic properties of a porous rock on the compressibility of the pore fluid, Geophysics, 40, 608-616. Cheng, C.H. and Toksoz, M.N. (1979), Inversion of seismic velocities for the pore aspect ratio spectrum of a rock, JGR, 84, 7533-7543.

Chun, K.-Y., G. A. Henderson, and J. Liu (2004), Temporal changes in P wave attenuation in the Loma Prieta rupture zone, JGR, 109, B02317, doi:10.1029/2003JB002498.

Eshelby, J.D. (1957), The determination of the elastic field for an elliptical inclusion and related problems, Proc. R. Soc. London, Ser. A, 241, 376-396.

Fredrich, J.T., Evans, B. et Wong, T-F. (1989), Micromechanics of the brittle to plastic transition in Carrara marble, JGR, 94, 4129-4145.

Gao, Y., and S. Crampin (2004), Observations of stress relaxation before earth- 
quakes, Geophys. J.Int., 157, 578-582.

Guéguen, Y. and A. Schubnel (2003), Elastic Wave Velocities and Permeability of Cracked Rocks, Tectonophysics, 370, 163-176.

Hadley, K. (1975), Azimuthal variation of dilatancy, JGR, 80, 4845-4850, 1975. Hazzard J.F. and Young R.P. (2004), Numerical investigation of induced cracking and seismic velocity changes in brittle rock, GRL, 31, Art. No. L01604.

Hudson, J.A. (1981), Wave speeds and attenuation of elastic waves in material containing cracks, Geophys. J. R. Astr. Soc., 64, 133-150.

Hudson, J.A. (1982), Overall properties of a cracked solid, Math. Proc. Cambridge Phil. Soc., 88, 371-384.

Hudson, J.A. (1986), A higher order approximation to the wave propagation constants for a cracked solids, Geophys. J. R. Astr. Soc., 87, 265-274.

Kachanov, M., (1994), Elastic solids with many cracks and related problems, Adv. Appl.Mech., 30 259-445.

Kern, H. (1978), The Effect of High Temperature and High Confining Pressure on Compressional Wave Velocities in Quartz Bearing and Quartz Free Igneous and Metamorphic Rocks, Tectonophysics, 44, 185-203.

Kern, H., Liu, B., and Popp, T. (1997), Relationship between Anisotropy of $P$ and $S$ wave velocities and Anisotropy of Attenuation in Serpentinite and Amphibolite, JGR, 102, 3051-3065.

King M.S. (2002), Elastic wave propagation in and permeability for rocks with multiple parallel fractures, Int. J. Rock Mech. Min. Sci., .39, 1033-1043.

Le Ravalec, M. and Guéguen, Y. (1996), High and low frequency elastic moduli 
for a saturated porous/cracked rock - Differential self consistent and poroelastic theories, Geophysics, 61 1080-1094.

Mavko,G. and Nur, A. (1975), Melt squirt in the asthenosphere, JGR, 80, 14441448.

Mavko,G. and Jizba, D. (1991),Estimating grain-scale fluid effects on velocity dispersion in rocks, Geophysics, 56, 1940-1949.

Mavko, G., Mukerji, T., and Dvorkin, J. (1998), in: The Rock Physics Handbook, Cambridge University Press, 329pp.

Miller, S. A. (2002), Properties of large ruptures and the dynamical influence of fluids on earthquakes and faulting, JGR, 107(B9), 2182, doi:10.1029/2000JB000032. Nasseri, M.H.B., Mohanty, B. and Robin P-Y. (2005), Characterization of microstructures and fracture toughness in .ve granitic rocks, International Journal of Rock Mechanics \& Mining Sciences, 42, 450Ü460.

Nishizawa, O. (1982), Seismic velocity anisotropy in a medium containing oriented cracks: Transverse isotropy case, J. Phys. Earth, 30, 331-347.

O'Connell, R., and Budiansky, B. (1974),Seismic velocities in dry and saturated rocks, JGR, 79, 5412-5426.

O'Connell, R., and Budiansky, B. (1977), Viscoelastic properties of fluid saturated cracked solids, JGR, 82, 5719-5736.

Sayers, C.M. and Kachanov, M. (1991), A simple technique for finding effective elastic constants of cracked solids for arbitrary crack orientation statistics, Int.J Solid Structures, 12, 81-97.

Sayers, C.M. and Kachanov, M. (1995), Microcrack induced elastic wave anisotropy 
of brittle rocks, JGR, 100, 4149-4156.

Schubnel, A. (2002), Mécanique de la dilatance et de la compaction des roches de la croûte, PhD thesis of Institut de Physique du Globe de Paris, 229pp.

Schubnel, A., Nishizawa, O., Masuda, K., Lei, X.J., Xue, Z. and Guéguen, Y. (2003), Velocity Measurements and Crack Density Determination during Wet Triaxial Experiments on Oshima and Toki Granites, Pure and Applied Geophysics, $160,869-887$.

Schubnel, A. and Y. Guéguen (2003), Dispersion and Anisotropy in Cracked Rocks, JGR, 108, 2101, doi:10.1029/2002JB001824.

Schubnel A., Fortin J., Burlini L. and Y. Guéguen (2005), Damage and recovery of calcite rocks Deformed in the Cataclastic Regime, Geological Society of London special publications, in press.

Simmons, G., and Brace,W.F. (1965), Comparison of Static and Dynamic Measurements of Compressibility of Rocks, JGR, 70, 5649-5656.

Simmons, G., Todd, T. and Balridge, W.S. (1975), Toward a quantitative relationship between elastic properties and cracks in low porosity rocks, AM. J. Sci., $275,318-345$.

Thomson B.D., Young R.P. and Lockner D.A. (2003), Acoustic emission monitoring of Westerly granite in Triaxial loading experiments: Results and Research direction, Lassonde Institute internal report, 68pp.

Walsh, J.B. (1965), The Effect of Cracks on the Compressibility of Rock, JGR, $70,381-389$.

Zamora, M., Pezzard, P.A. et Ito, H. (1999), Anisotropy of elastic properties of 
granites from the Hirabayashi borehole, Japan, internal note, GSJ/USGS. 


\section{Appendix I: Elastic deformation of a single penny-shaped crack}

For a single penny shaped crack under a uniform normal load $p$ or shear stress $\tau$, the elastic normal and shear displacements of the crack surfaces are not equal (and thus there can be a non-collinearity between the stress and displacement vectors). Such elastic displacements $<\mathbf{b}>$ can be written respectively as (Kachanov [1994]):

$$
<\mathbf{b}>= \begin{cases}<b_{n}>= & \frac{16\left(1-\nu^{2}\right) c}{3 \pi E} p \\ <b_{\tau}>= & \frac{16\left(1-\nu^{2}\right) c}{3 \pi(1-\nu / 2) E} \tau\end{cases}
$$

where $c$ is the crack radius, $E$ and $\nu$ the matrix Young's modulus and Poisson ratio respectively. In the case of a crack filled up with fluid, an applied stress on the crack surface will generate a pore pressure variation $\Delta p_{f}$. Considering the crack aspect ratio $\zeta=w / c$, and assuming that $w<<c$, the volume variation of the crack is mainly related to a change in its aperture. Thus the total traction applied on the crack surface is $\mathbf{n} . \sigma \cdot \mathbf{n}+\Delta p_{f}$. From equation (12), one obtains:

$$
<\Delta b_{n}>=\frac{16\left(1-\nu^{2}\right) c}{3 \pi E}\left(\mathbf{n} \cdot \sigma \cdot \mathbf{n}+\Delta p_{f}\right)
$$

Considering that the fluid mass is constant, the variation in fluid density, volume and aperture are linked:

$$
\frac{\Delta \varrho}{\varrho_{o}}=-\frac{\Delta V}{V_{o}}=\frac{-<\Delta w>}{<w>}=-\frac{\left.<\Delta b_{n}\right\rangle}{<w>}
$$

Remembering that $\Delta p_{f}=-K_{f} \frac{\Delta \varrho}{\varrho_{o}}$, the crack normal compliance $B_{N}$ is such that (Kachanov [1994]):

$$
\frac{B_{N}}{B_{T}}=\left(1-\frac{\nu_{0}}{2}\right) \frac{\delta}{1+\delta}
$$


where $\delta$ characterizes the coupling between the stress and the fluid pressure, and determines the fluid impact on the effective crack compliance :

$$
\delta=\chi^{-1} \frac{E_{0} \zeta}{K_{f}}
$$

where $K_{f}$ is the fluid bulk modulus. $\zeta$ is the average aspect ratio of the crack defined as $\zeta=\frac{\langle w\rangle}{a}$, where $\langle w\rangle$ is the average crack aperture, and $\chi$ is a geometrical factor: $\chi=16 \frac{1-\nu_{0}^{2}}{3 \pi}$ that describes the penny-shaped geometry of the crack. 


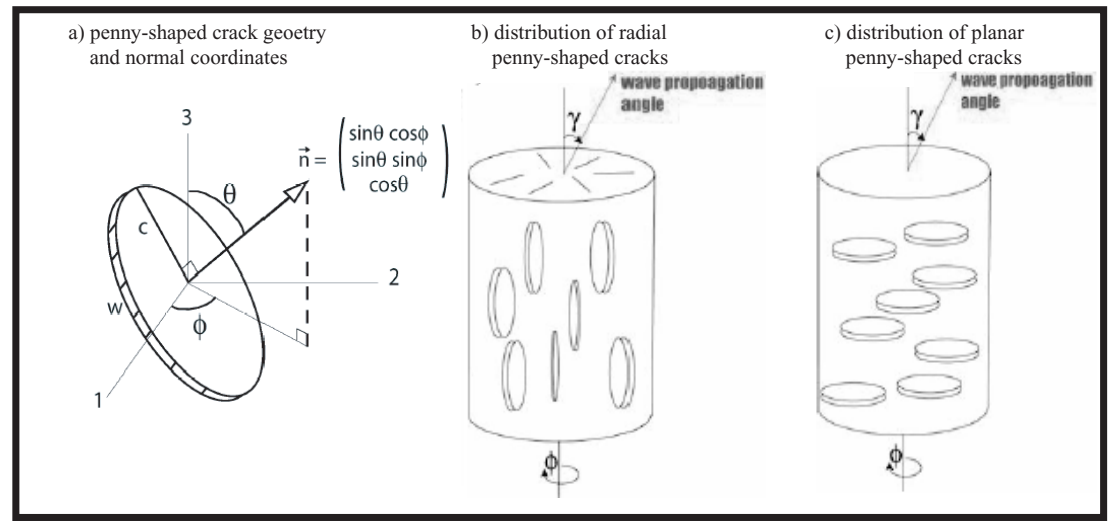

Figure 1: a) Geometry, conventions and coordinates of a 3-D penny-shaped crack. The two crack distributions considered here: a) the case of planar penny shaped cracks, b) the case of radial penny shaped cracks. 


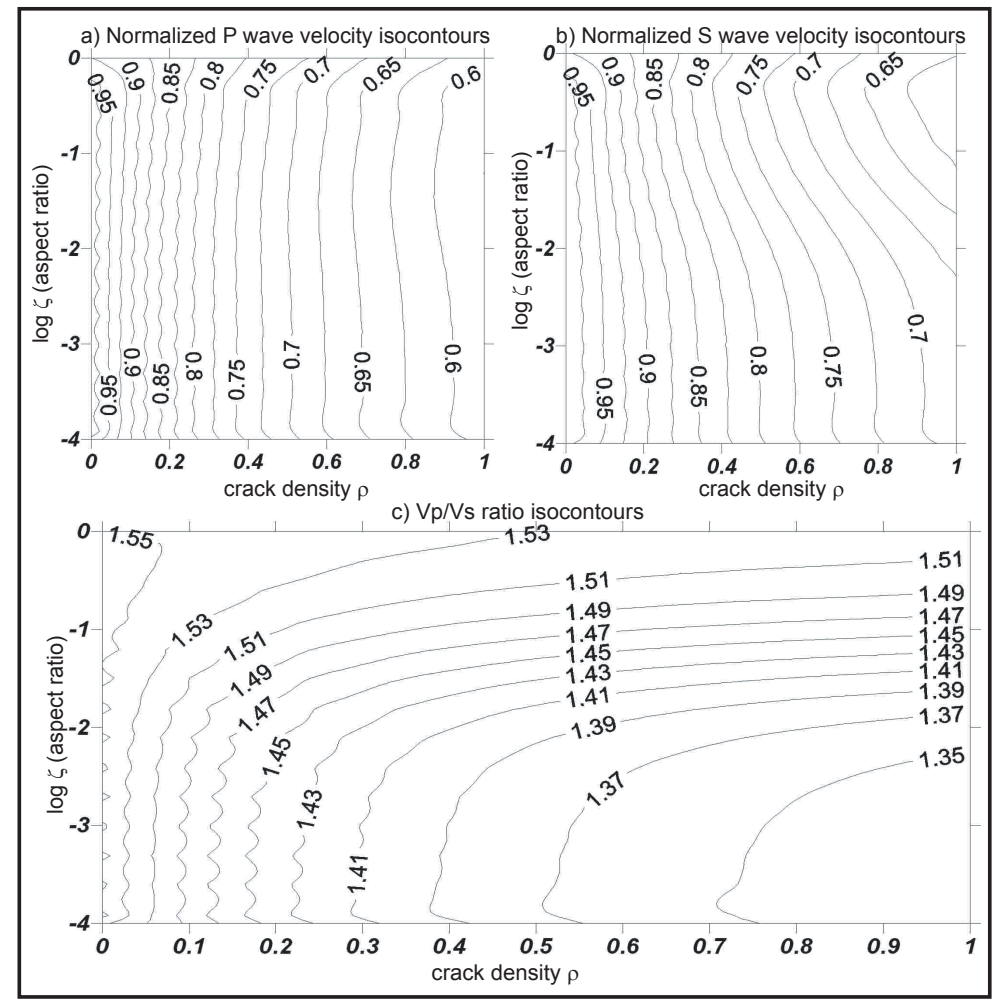

Figure 2: Isotropic case: a) and b) normalized $\mathrm{P}$ and $\mathrm{S}$ wave velocity isocontours as a function of both crack density and the aspect ratio. Velocities were normalized to the crack free velocities (i.e. $\rho=0$ ) to remove bulk density effects. c) evolution of the $\mathrm{Vp} / \mathrm{Vs}$ ratio as function of both crack density and aspect ratio. 


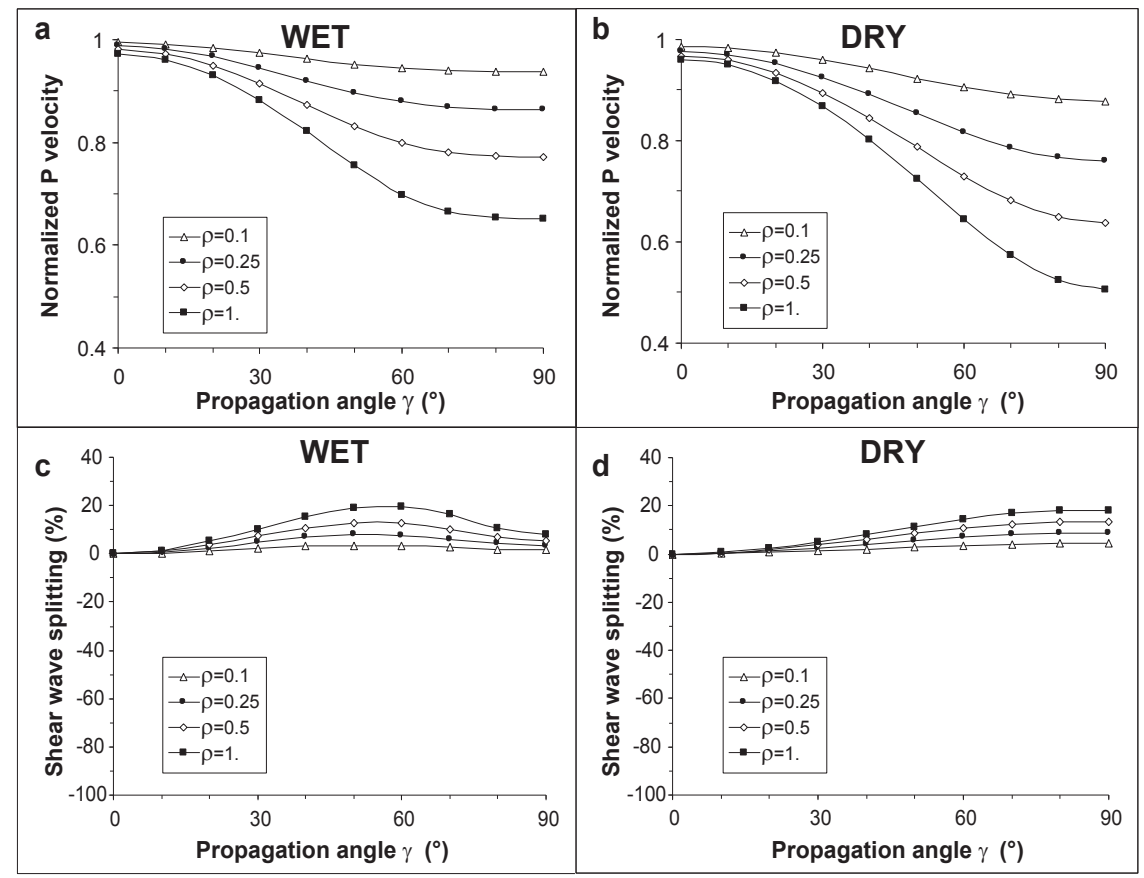

Figure 3: Case of radial cracks (figure 1b): Velocities were normalized to the crack free velocities (i.e. $\rho=0$ ) to remove bulk density effects. Results are displayed for crack densities equal to 0.1 (open triangles), 0.25 (plain circles), 0.5 (open diamonds) and 1 (plain squares). $\mathrm{a}$ and $\mathrm{b}$ show the normalized $\mathrm{P}$ wave velocity as a function of the direction of propagation $\gamma$ in the wet and dry cases respectively. $\mathrm{c}$ and $\mathrm{d}$ show the shear wave splitting (or birefringence) as a function of the direction of propagation $\gamma$ in the wet and dry cases respectively. 


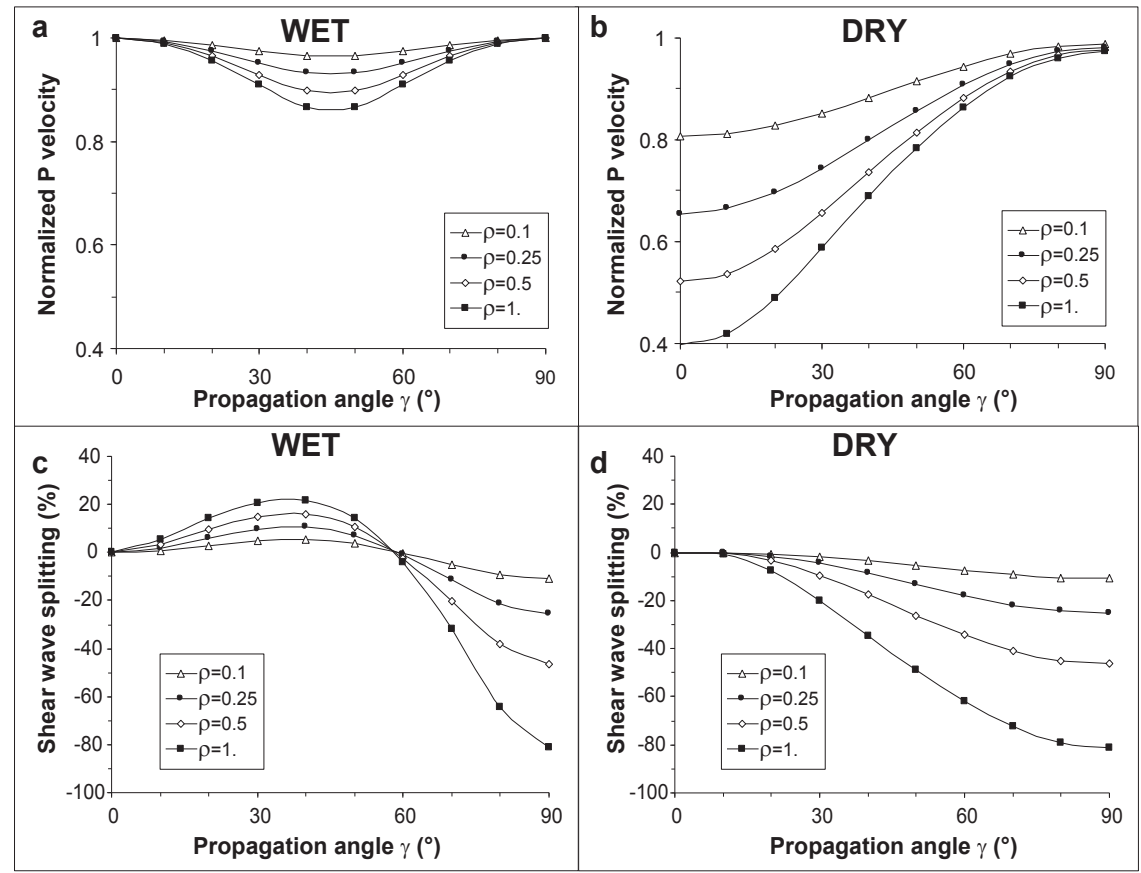

Figure 4: Case of planar cracks (figure 1c): Velocities were normalized to the crack free velocities (i.e. $\rho=0$ ) to remove bulk density effects. Results are displayed for crack densities equal to 0.1 (open triangles), 0.25 (plain circles), 0.5 (open diamonds) and 1 (plain squares). a and $\mathrm{b}$ show the normalized $\mathrm{P}$ wave velocity as a function of the direction of propagation $\gamma$ in the wet and dry cases respectively. $\mathrm{c}$ and $\mathrm{d}$ show the shear wave splitting (or birefringence) as a function of the direction of propagation $\gamma$ in the wet and dry cases respectively. 


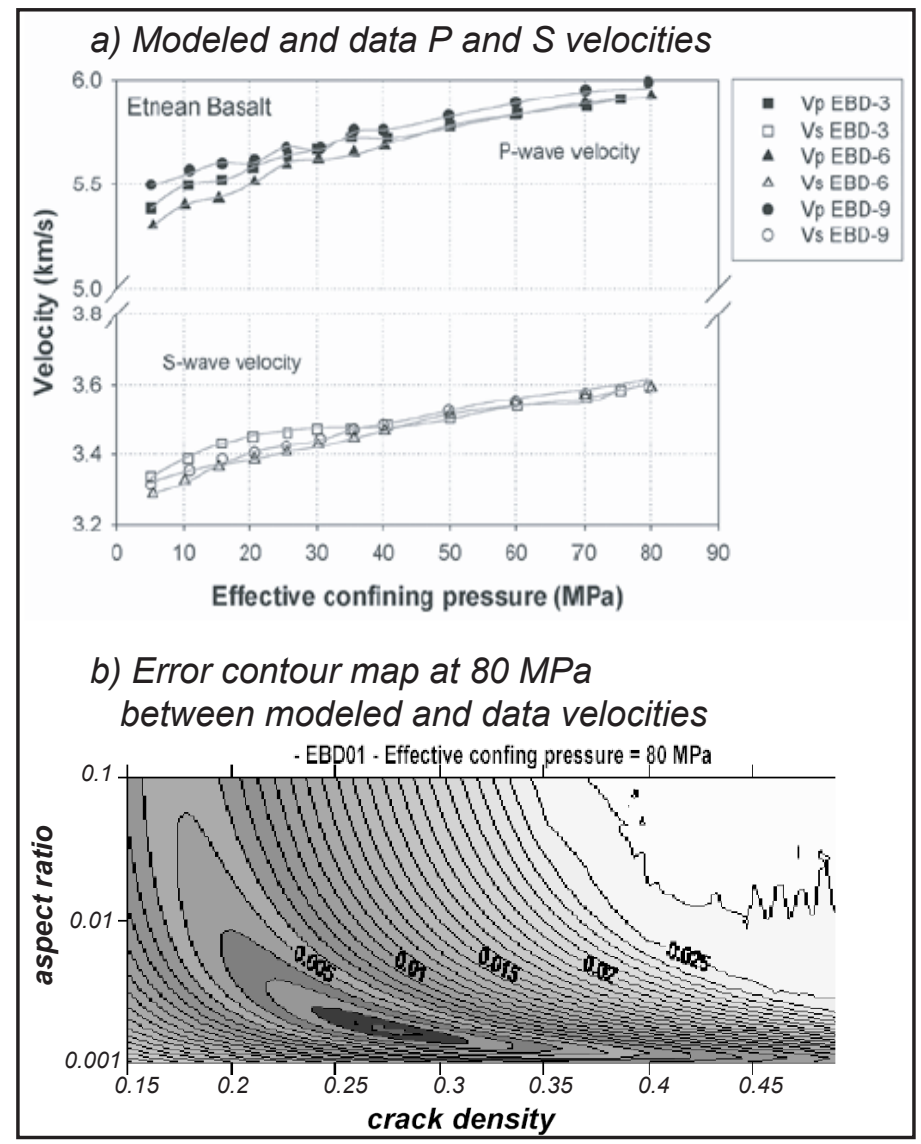

Figure 5: a) Modeled (solid lines) P and S velocities in Etna basalt compared to the experimental measurements (dots). The effective confining pressure was calculated as $P_{c}-P_{p}$ where $P_{c}$ is the confining pressure and $P_{p}$ the pore pressure. b) Error contour map between modelled and data (P and S) velocities in the case of sample EBD01 at $80 M P a$ confining pressure. Error contours are displayed as a function of crack density and aspect ratio respectively. Darker areas represent lower errors. 


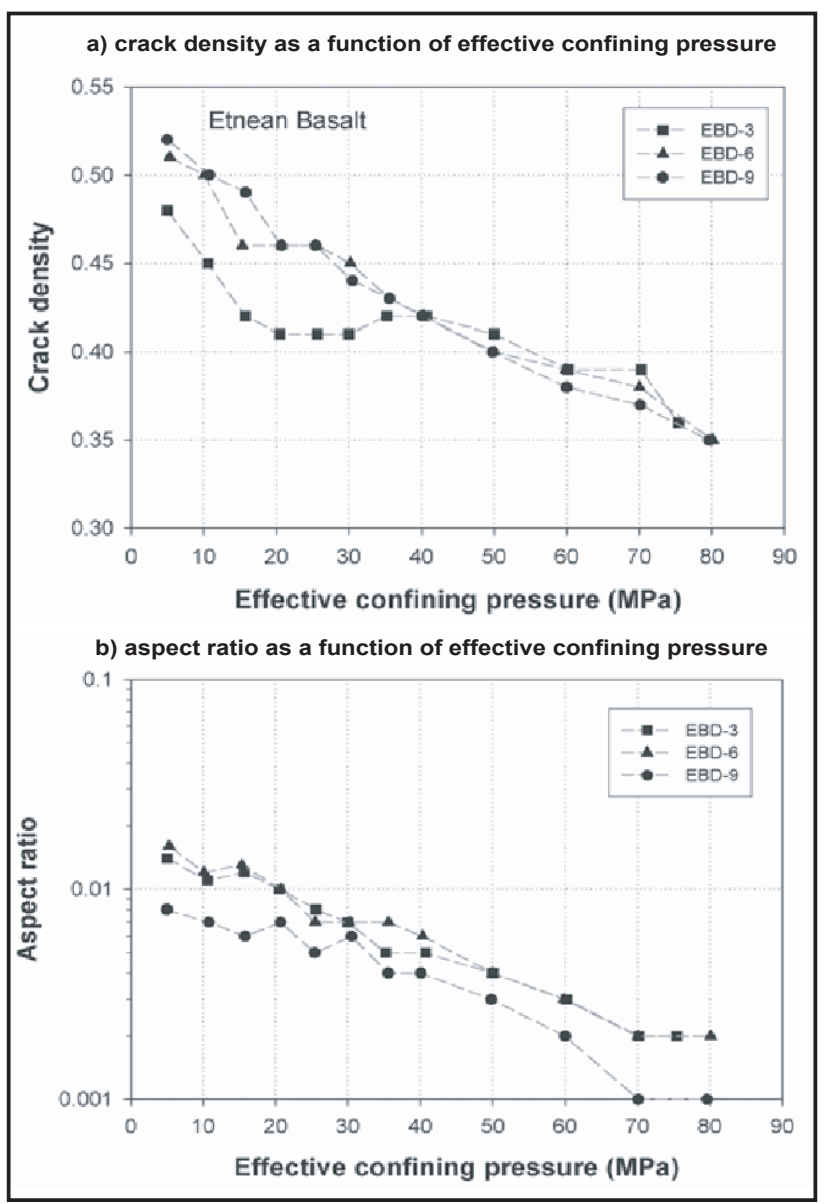

Figure 6: Evolution of crack density (a) and aspect ratio (b) as a function of confining pressure. Both crack density and aspect ratio were inverted from experimental data presented on figure 5 and using equations (5) and (6). Matrix elastic parameters were taken as $E=100 G P a$ and $\nu=0,22$ (i.e. crack free elastic wave velocities equal to $V_{p}=6400 \mathrm{~m} / \mathrm{s}$ and $V_{s}=3750 \mathrm{~m} / \mathrm{s}$ ) 


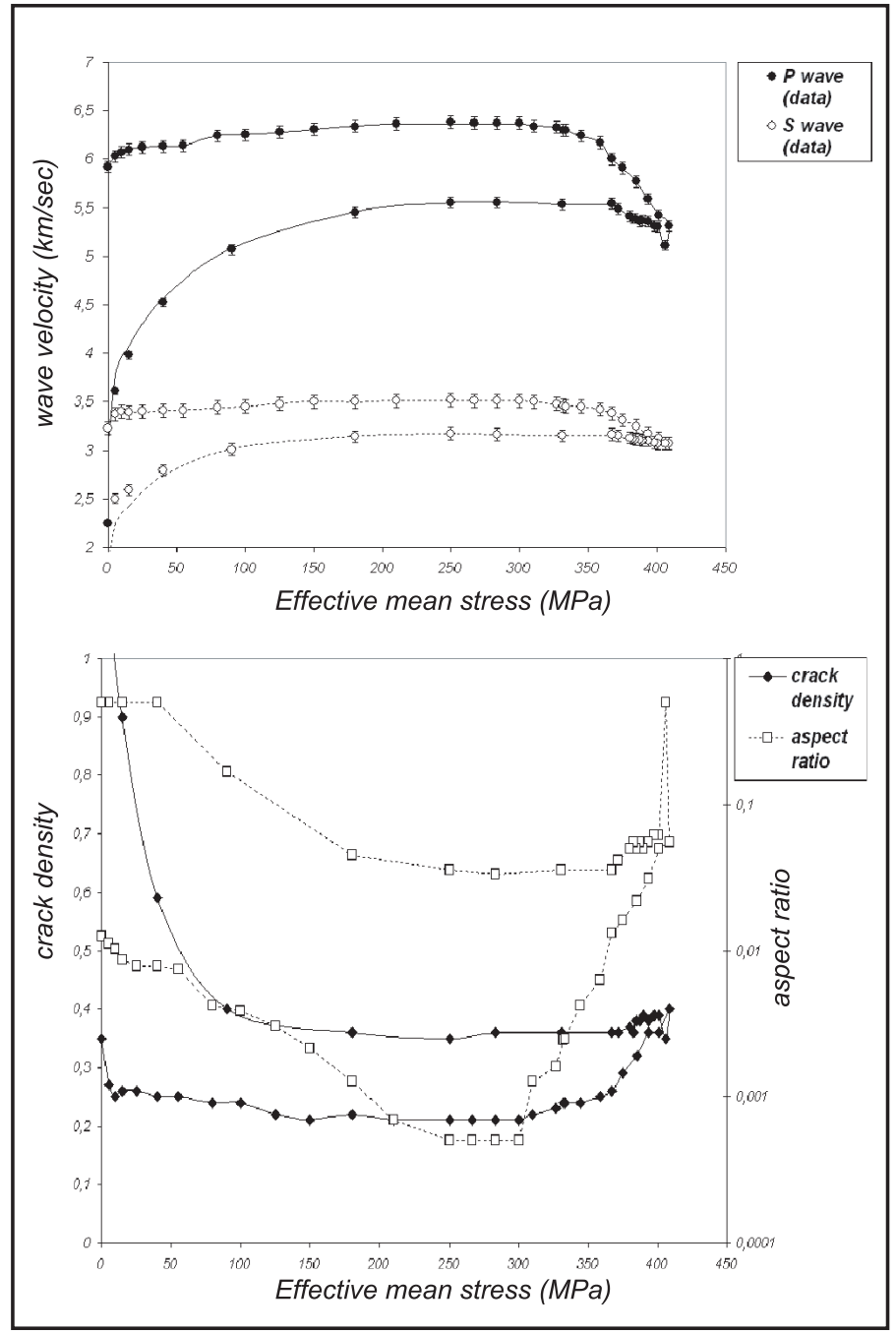

Figure 7: a) Modelled (lines) and data (dots) $\mathrm{P}$ and $\mathrm{S}$ wave velocities as a function of effective mean stress. Experiment was performed at $P c=260 \mathrm{MPa}$ confining pressure and $P p=10 M P a$. Model crack-free parameters were taken as $E=100 G P a$ and $\nu=0.32$. b) Evolution of crack density and aspect ratio as a function of effective mean stress. Both crack density and aspect ratio were inverted from experimental data presented on figure a and using equations (5) and (6). 


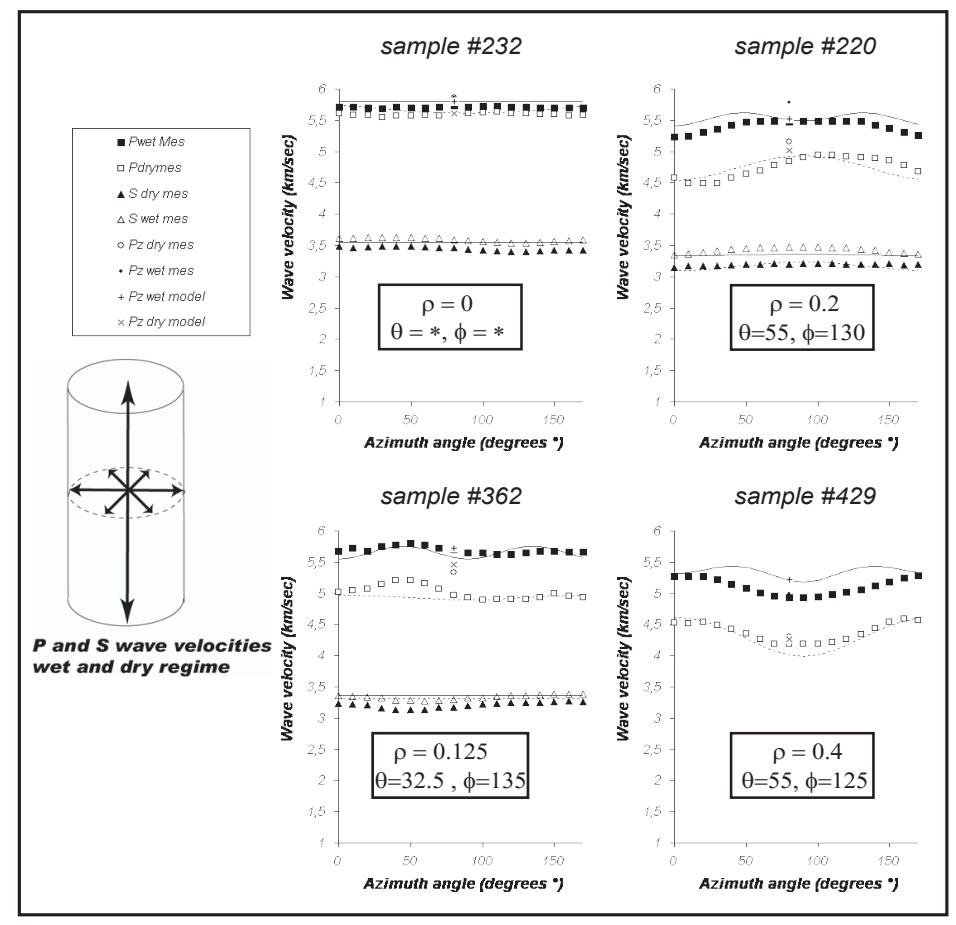

Figure 8: $\mathrm{P}$ and $\mathrm{S}$ wave velocities measurements in dry (empty symbols) and wet conditions (plain symbols). Measurements were performed at room pressure by Zamora et al. 1999. P wave were measured along the vertical axis and along 18 different directions in the horizontal plane; $\mathrm{S}$ waves propagating along the vertical axis were measured along 18 different horizontal polarizations. Experimental data (dots) obtained on 4 different samples, retrieved at 220, 232, 362 and 429 meters respectively is present 29 . Modeled velocities for each of these samples are represented by solid and dashed line. Crack free elastic parameters were taken as equal to that of sample $232\left(E_{o}=85 G P a\right.$ and $\left.\nu_{o}=0.25\right)$. The inversion outputs both the crack density $\rho$ and the crack fabric orientation 


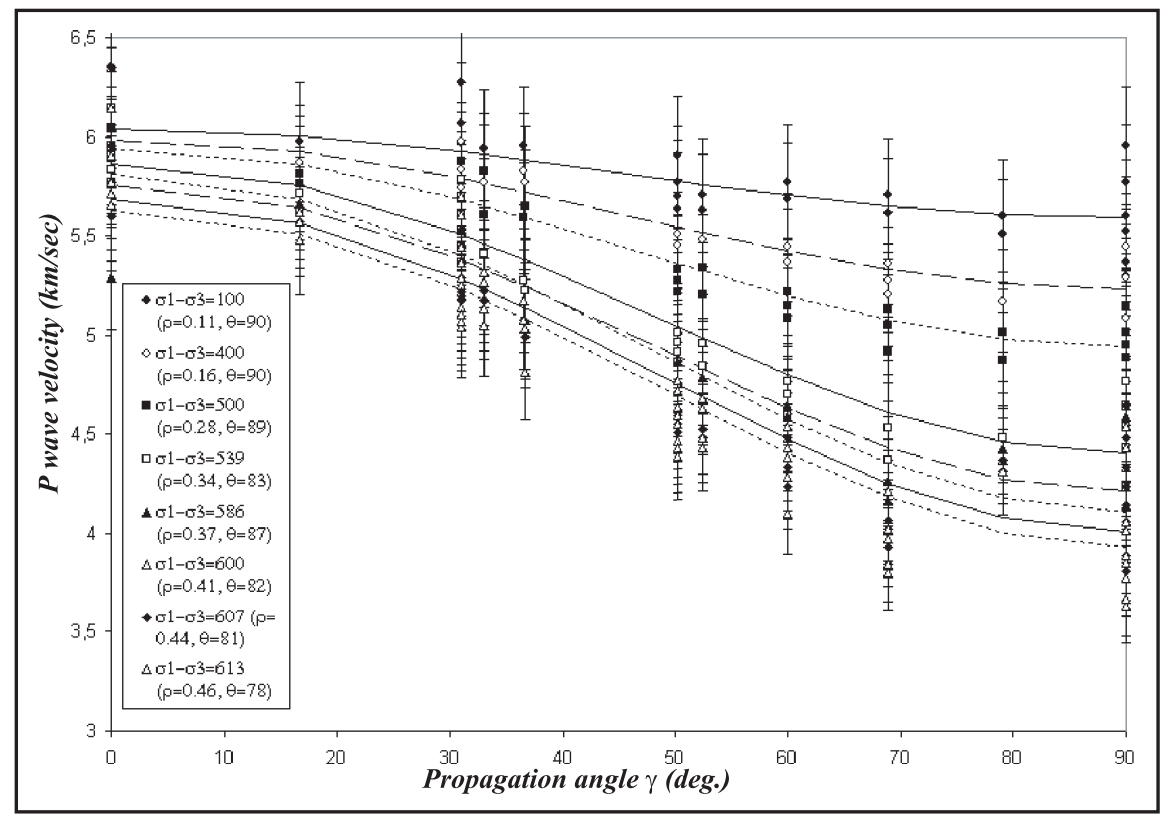

Figure 9: Modelled (lines) and data $\mathrm{P}$ wave velocity field as a function of stress and raypath angle (from vertical). Experiment was performed at $P c=50 \mathrm{MPa}$ confining pressure in dry conditions. Model crack-free parameters were taken as $E=85 G P a$ and $\nu=0.25$. At each shear stress step, modelled crack density and average crack fabric dip are marked on the legend. 


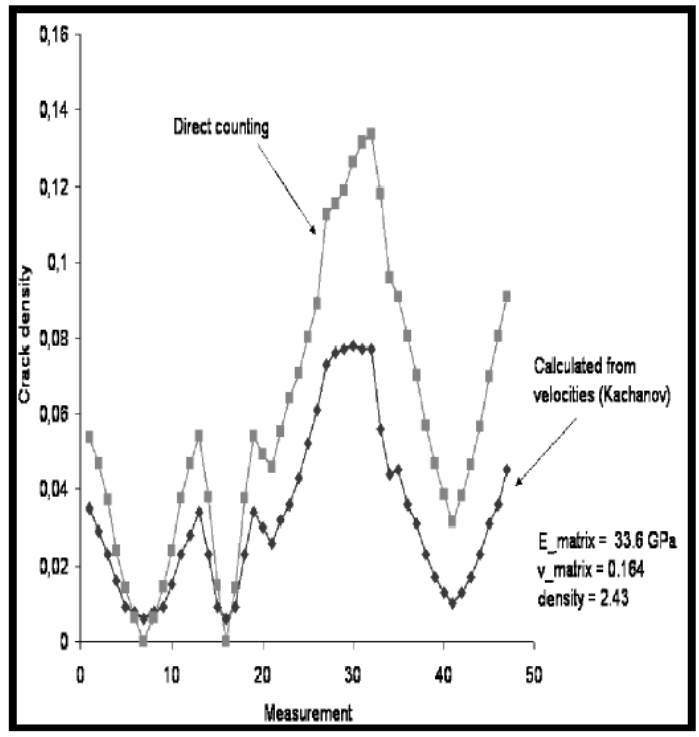

Figure 10: Comparison between PFC model directly observed crack density (crack radii were taken as equal as particle's) and Kachanov's model inversion results for Crossland sandstone. 